\title{
微矿分离: 煤炭清洁化与土壤改良的新契机
}

吴昌宁 ${ }^{1,2,3}$, 翁力 ${ }^{1,2}$, 李俊国 ${ }^{1,2,3^{*}}$, 贾吴霖 ${ }^{1}$, 王欣 ${ }^{1}$, 陈加利 ${ }^{1}$, 黄伟 ${ }^{1}$, 胡顺轩 ${ }^{1}$, 曹道帆 ${ }^{1}$, 周宝楠 ${ }^{1}$, 楚长青 ${ }^{2}$, 赵冰龙 ${ }^{3}$, 刘科 $1,2,3^{*}$

1. 南方科技大学化学系, 深圳 518055 ;

2. 南方科技大学前沿与交叉科学研究院, 清洁能源研究院, 深圳 518055 ;

3. 南方科技大学创新创业学院, 深圳低质煤综合利用工程研究中心, 深圳 518055

*联系人, E-mail: lijg@sustech.edu.cn; liuk@sustech.edu.cn

2021-01-16 收稿, 2021-04-01 修回, 2021-05-06 接受, 2021-05-27 网络版发表

广东省创新创业团队项目(2016ZT06N532)、深圳市高层次人才创新创业计划(KYTDPT20181011104002，KQTD20180411143418361)、深圳市 绿色低碳产业发展专项资金(XMHT20190203001)、深圳清洁能源研究院项目(CERIKY-2019-003)、深圳燃气-南科大联合研发项目 (S1802017)、吕梁市政府-南科大绿色清洁能源联合实验室项目(TO2005006)和北京热力-南科大创新创业学院联合实验室项目(TO2005002) 资助

摘要报道一种新型的矿物分离技术。该技术集成了磨矿、分离、颗粒级配和土壤改良技术, 是结合微纳米科学 与材料加工技术等开发的一种多学科交叉的能源化工创新性技术. 利用此技术可以高效地选择性脱除含碳材料源 中的微量元素及矿物质, 制备出清洁固体燃料(clean solid fuel, CSF)、清洁类液体燃料(pseudo liquid fuel, PLF)以及 土壤改良矿物质(soil remediation minerals, SRM), 简称为微矿分离(RMS)技术. 该技术主要包括4个工艺步骤: (1) 基 于矿物嵌布形态解构, 采用流体磨实现碳质组分与无机组分的高效解离; (2) 采用颗粒表面改性、多相流界面调控 及专用助剂等将碳质颗粒和无机颗粒高效分离; (3) 分离之后形成的碳质颗粒流, 脱水制成CSF, 或者进入PLF制备 单元，同时无机颗粒流经脱水制成SRM; (4) 采用多峰级配将碳质颗粒制成高浓度PLF. RMS工艺技术经小试和工 业放大试验，已完成工业放大验证，可燃体回收率大于 $80 \%$ 的同时CSF灰分小于 $7 \%$. SRM制成的天然矿物质肥料和 土壤改良剂，可有效缓解表土流失问题，提高植被及作物产量，通过植物及土壤增量固碳，进而达到碳中和目的. RMS技术可生产低成本的清洁燃料并可实现煤基固废的资源化利用，为煤炭的高效清洁化利用和 $\mathrm{CO}_{2}$ 减排提供了 可靠的途径.

关键词 矿物分离, 清洁固体燃料, 类液体燃料, 土壤改良矿物质, 碳中和

化石能源的不合理使用给人类赖以生存的自然环 境带来了严重的危害. 由煤炭开采与利用带来的环境 污染、表土流失和土地退化，已成为一个全球性问题， 而这些问题在中国尤其突出. 中国2019年消耗煤炭量 近38.5亿吨(http://www.bp.com/statisticalreview). 全国 电力的 $64.7 \%$ 来自燃煤发电, 占比很高 ${ }^{[1]}$. 据国务院发 展研究中心 2017 年的统计数据, 民用生活燃煤约 2.34
亿吨、小锅炉燃煤约2.2亿吨、工业小窑炉燃煤约 2.36 亿吨、其他未完全统计的散煤约 0.6 亿吨，全国散煤消 费总量约为 7.5 亿吨. 这是中国空气污染和雾䨪的头号 来源，在3 5年内压缩至 5 亿吨的目标非常具有挑战 性 $^{[2-4]}$. 在新型冠状病毒疫情防控期间, 社会活动水平 大幅度降低, 污染物排放也大幅度降低, 北方地区却仍 然出现重污染天气. 据S cience 最新报道, 新型冠状病毒

引用格式: 吴昌宁, 翁力, 李俊国, 等. 微矿分离: 煤炭清洁化与土壤改良的新契机. 科学通报, 2021, 66: 3352-3364 Wu C N, Weng L, Li J G, et al. A novel mineral separation process: New opportunity for clean coal utilization and soil remediation (in Chinese). Chin Sci Bull, 2021, 66: 3352-3364, doi: 10.1360/TB-2021-0067 
疫情期间的空气污染数据证实了煤炭的不正当使用是 关键污染源 ${ }^{[5]}$. 给每台中小型燃煤锅炉配备脱硫脱硝设 施，如选择性催化还原(selective catalytic reduction, $\mathrm{SCR}$ ), 其成本过高，基本无法实施. 同时，中国的天然 气供应能力十分有限, 总当量仅约为 2.2 亿吨标准煤, 难 以将天然气作为中小型燃煤锅炉的替代燃料. 因此, 对 中国而言, 在燃烧或转化前将煤炭处理干净是一种必 然选择, 也是有效改善中国雾䨪状况的一种低成本解 决方案, 同时也可对大型煤电与煤转化过程进行人料 清洁化改进, 使其操作费用与环保成本显著降低.

同时，我国煤炭资源中有 $40 \%$ 以上为低质煤(包括 劣质煤、洗选中煤、煤泥等), 低质煤的高效清洁化和 资源化利用是行业一大难题. 以煤泥为例，年产量近2 亿吨，但普遍存在热值低、燃烧洁净度低、再利用难 度高、运输成本高等问题，亟待提高加工利用技术水 平 ${ }^{[6]}$. 此外, 煤矸石是煤炭生产和加工过程中产生的固 体废弃物，其排放量相当于原煤产量的 $10 \%$ 左右，目前 已累计堆存 30 多亿吨, 形成䂥石山1500余座, 合计占地 约 1.2 万公顷 $\left(1\right.$ 公顷 $\left.=1 \times 10^{4} \mathrm{~m}^{2}\right)$, 占中国工业固体废物排 放总量的 $40 \%$ 以上 ${ }^{[7]}$. 煤矸石因原矿不同, 其主要成分 和含量也不尽相同, 主要含有碳质燃料、腐殖酸、 硅、铝、钲、镁、钾、嗍、铁以及多种微量元素。煤 研石弃置不用，除占用大片土地外，还会自燃发生火 灾，煤矸石中逸出或浸出的硫化物会污染大气、农田 和水体等. 煤研石主要被用于制备矸石水泥、混凝土 轻质骨料、耐火砖等, 还可与煤混烧发电, 从煤灰中提 取氯化铝、氧化铝及贵重稀有金属等. 为此, 这一领域 的先进技术开发具有非常重要的环保和社会价值.

第二次世界大战之后，硝酸铵等化肥大量投人使 用, 显著提高了作物的产量, 但同时也影响了土壤结构 及物质组成. 土壤板结问题日趋严重, 其主要原因包括 钙离子固化、腐殖质分解加速、土壤酸化加剧、微生 物/矿物质平衡失调等. 土壤中的中微量矿质元素对植 物蔬果生长发育、抗性生理和营养口味均起着重要作 用. 早年间使用的化肥(如硝酸铵)主要由强酸(硝酸)和 弱碱(氢氧化铵)组成. 农业措施中氮肥的施用是加速土 壤酸化的主要原因. 随着铵态氮的吸收转化和硝态氮 的淋失, 土壤逐渐发生酸化, 导致表土中的微量元素和 矿物质被浸出、流失. 经过几十年的作用, 土壤中作物 所需的中微量元素含量逐渐降低; 并且, 化肥使植物快 速生长, 导致土壤中有限的微量元素来不及被植物有 效吸收，这也解释了为什么长期使用化肥之后导致食
物的口味变差. 如图1所示，随着人工合成化肥和农药 在农业中的进一步使用，两类问题凸显：(1) 酸性浸出 和过度耕种导致的土壤中微量元素及矿物质流失 ${ }^{[8]}$; （2）酸性环境下土壤团聚体受破坏以及微生物难以生 存导致日趋严重的土壤板结, 限制了作物的生长. 根据 美国农业部报道, 从19世纪50年代开始, 表土中的矿物 质元素流失比例高达 $80 \%$, 并且随着化肥的长期使用, 美国疾病发生率高速增长并呈现失控状态，人体健康 程度、精神疾病治愈率及生活品质不断下降 ${ }^{[9]}$. 近期 的研究表明，尽管随着全球 $\mathrm{CO}_{2}$ 排放量持续增大，可促 进植物增长的 $\mathrm{CO}_{2}$ 量增加了, 但自 1982 年以来, 全球植 物因为水和营养物质的减少而导致了 $\mathrm{CO}_{2}$ 对植物的促 生长效应衰减了 $50 \%$. 这一变化将会对地球碳循环和气 候造成非常严重的影响 ${ }^{[10]}$.

为了解决由土壤退化引发的可食用植物营养质量 问题, 有不少研究人员尝试通过优质野生植物品种交 叉培育与基因改造来解决粮食安全问题，但要实现消 除基因改造过程风险、成本可控以及大规模推广仍是 一个十分漫长的过程 ${ }^{8}$. 这要求我们从另外一个视角来 探讨解决土壤退化和粮食安全问题的方法途径.

植物中的可燃部分是由光合作用合成的，剩下的 不可燃部分是来自土壤中的矿物质. 煤由远古时期的 植物通过表土覆盖所生成, 尽管这些大树被埋藏在地 下几百万年, 但是这些矿物质没有消失. 供远古时期植 物生长的土壤中的矿物质及微量元素也参与成煤过程, 并作为主要矿物质来源被保存于煤层中 ${ }^{[11]}$. 煤中的矿 物在燃烧之前分离出来, 通过深人探讨并理解其中矿 物质及微量元素所经历的化学及生物过程，则可能通

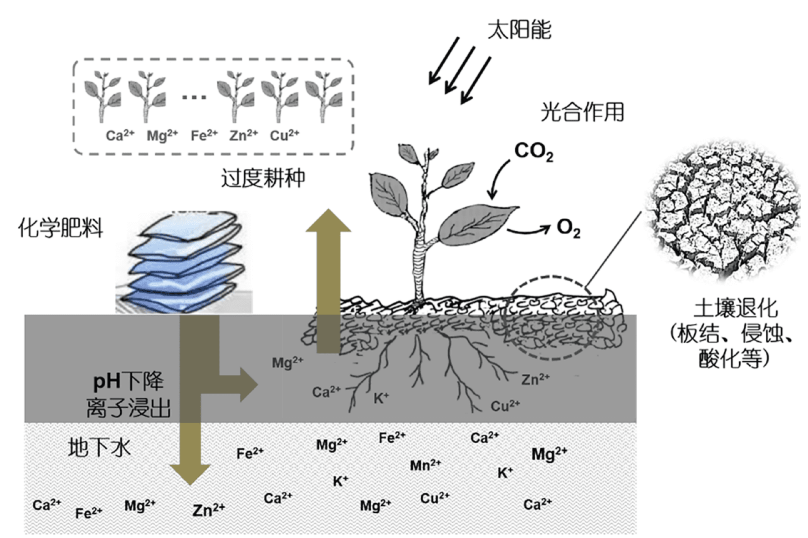

图 1 (网络版彩色)大量酸性化学肥料使用导致土壤中矿物质流失 过程示意图

Figure 1 (Color online) Schematic diagram of mineral loss from soil caused by overutilization of acid chemical fertilizers 
过一系列物理、化学和生物过程将煤中的矿物质及微 量元素成分返回其原始状态，这些矿物质成分可以用 来制备天然的土壤改良剂(soil remediation amendment, SRA). 值得指出的是, 如果煤在锅炉或气化炉中经历高 温燃烧或转化，煤中的矿物质由于氧化和聚团作用将 被钝化或失活，进而导致燃烧后的矿物质较难用来制 备天然的SRA.

总而言之, 要解决上述的能源和环境问题, 亟须开 发高效、低成本的煤与煤基固废清洁化/资源化利用技 术. 此类技术应在煤与煤䂥石利用前即将其洁净化加 工处理，并可同时制备清洁固体燃料(clean solid fuel, CSF)及天然矿源SRA.

\section{1 微矿分离工艺技术}

本课题组 ${ }^{[12,13]}$ 经过多年的研究积累, 针对煤炭及 煤基固废等含碳材料源开发了一项新型的分离提质工 艺技术. 该技术将含碳材料源在燃烧/转化前湿法粉碎 解离到微米级粒度, 并经颗粒表面改性、多相流界面 调控之后将含碳材料源中的微量元素及矿物质分离出 去, 获得清洁碳粒悬浮液(clean particulate suspension, CPS). 该清洁碳粒可制成CSF或者与级配颗粒群共同 分散在有机液体及水中，制成清洁的类液体燃料(pseu- do liquid fuel, PLF); 分离出的微细尺度、未经地表污 染并具有土壤改良功能的矿物质(soil remediation minerals, SRM)可制成天然矿物质肥料(natural mineral fertilizer, NMF)和SRA. 该技术是颗粒粒度控制、微纳 米技术、材料加工、化工分离和系统控制多学科交叉 的一个创新性应用成果，因其可选择性脱除含碳材料 源中的微量元素及矿物质, 简称为“微矿分离”(RevTech minerals separation, RMS)技术. RMS技术的主要产品方 案, 如图2所示.

\section{1 工艺流程简介}

RMS技术的工艺流程, 详见图3(a). 以原煤物料为 例, 湿法介质为水, 主要包括以下步骤: (1) 根据来料的 工艺矿物学特性, 选择性地破磨, 制成 $0.5 \mathrm{~mm}$ 以下细颗 粒. (2) 基于矿物嵌布形态表征结果, 确定微粒碳质组 分-无机组分解离的优选粒度, 通过高效解离磨, 将细颗 粒进一步制成微细颗粒，实现碳质组分与无机组分的 高效解离. (3) 在制好的微细煤浆中, 添加专用助剂, 并 辅以剪切活化、定位空化等手段以改变颗粒的表面性 能，在专门设计的微纳分离塔中辅以微纳米气泡、选 择性絮团、气液固流型调控等手段实现碳质、无机颗 粒的高效分离, 获得富含碳质颗粒的CPS物流和无机颗

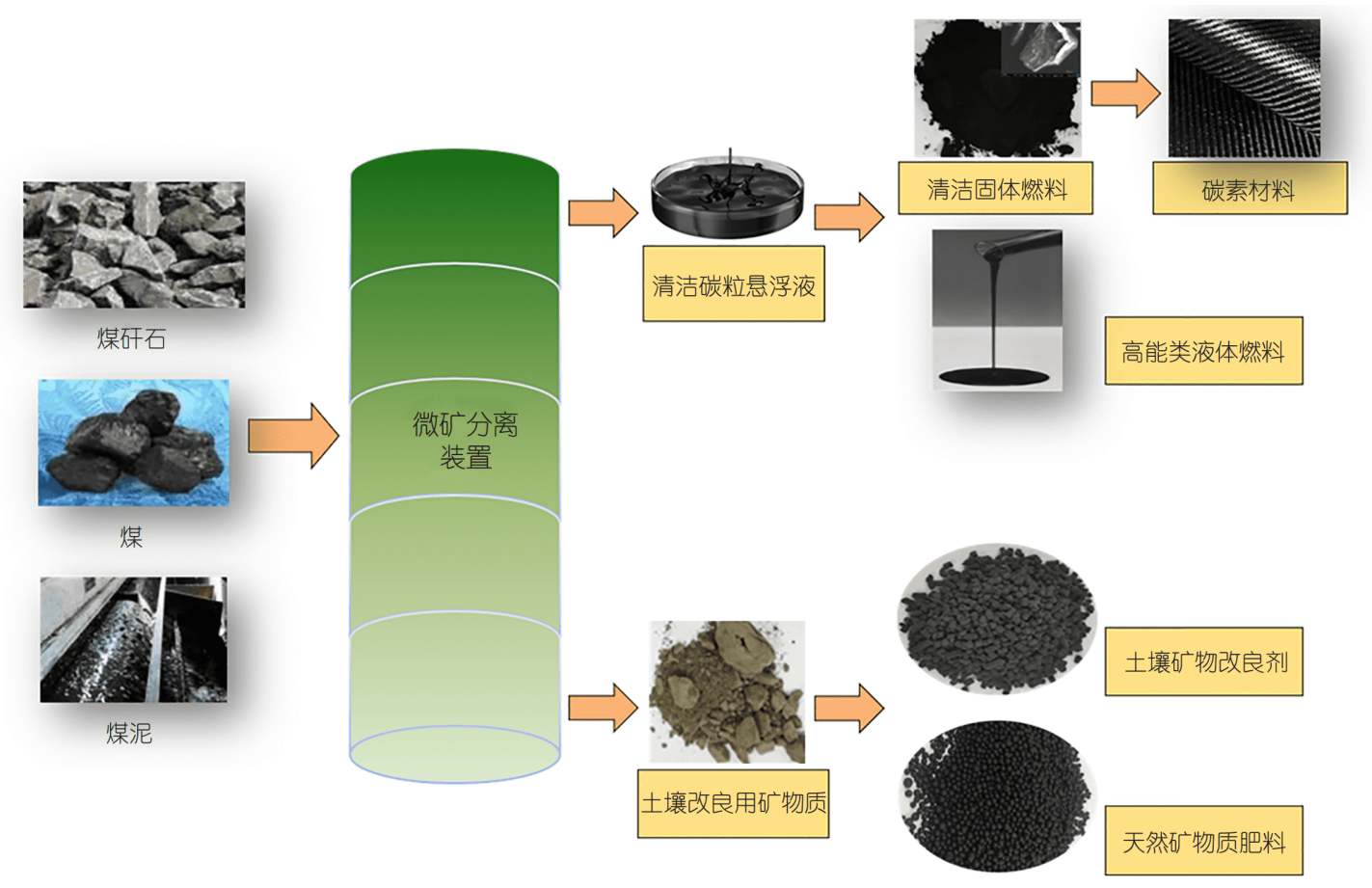

图 2 微矿分离工艺的主要产品方案

Figure 2 Schematic diagram of RMS process related products 


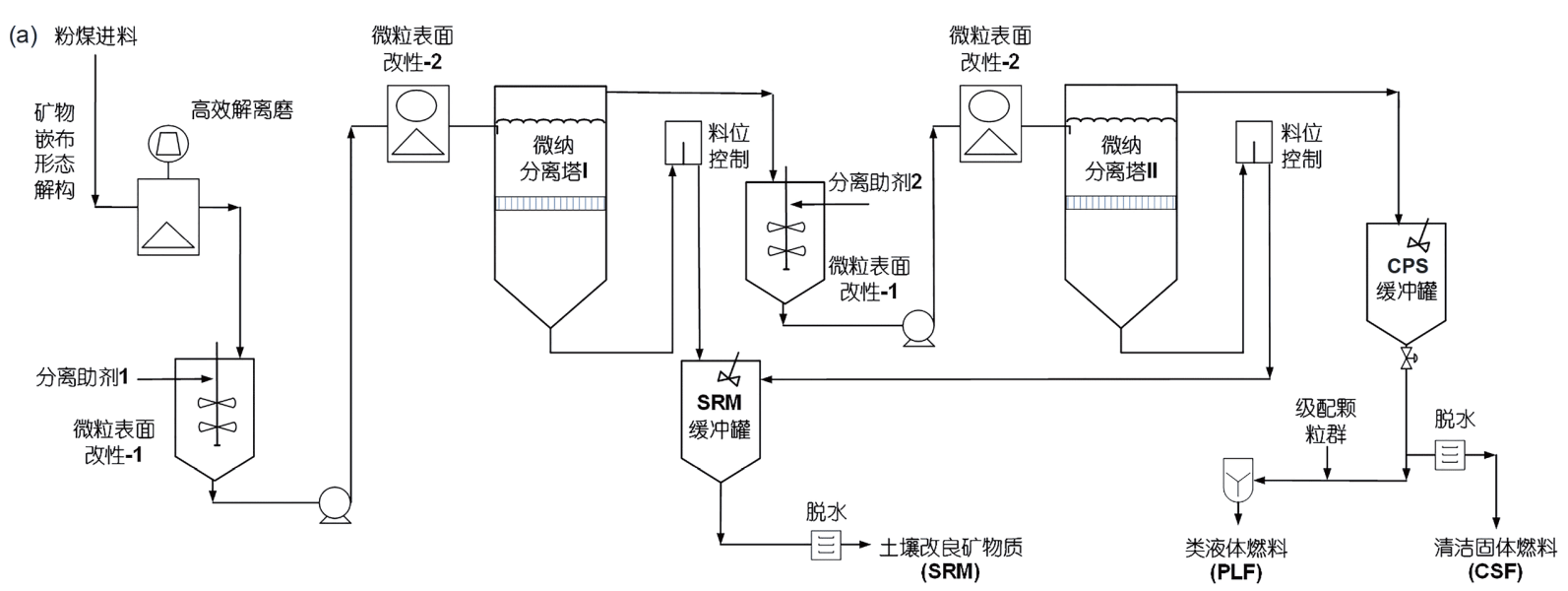

(b)

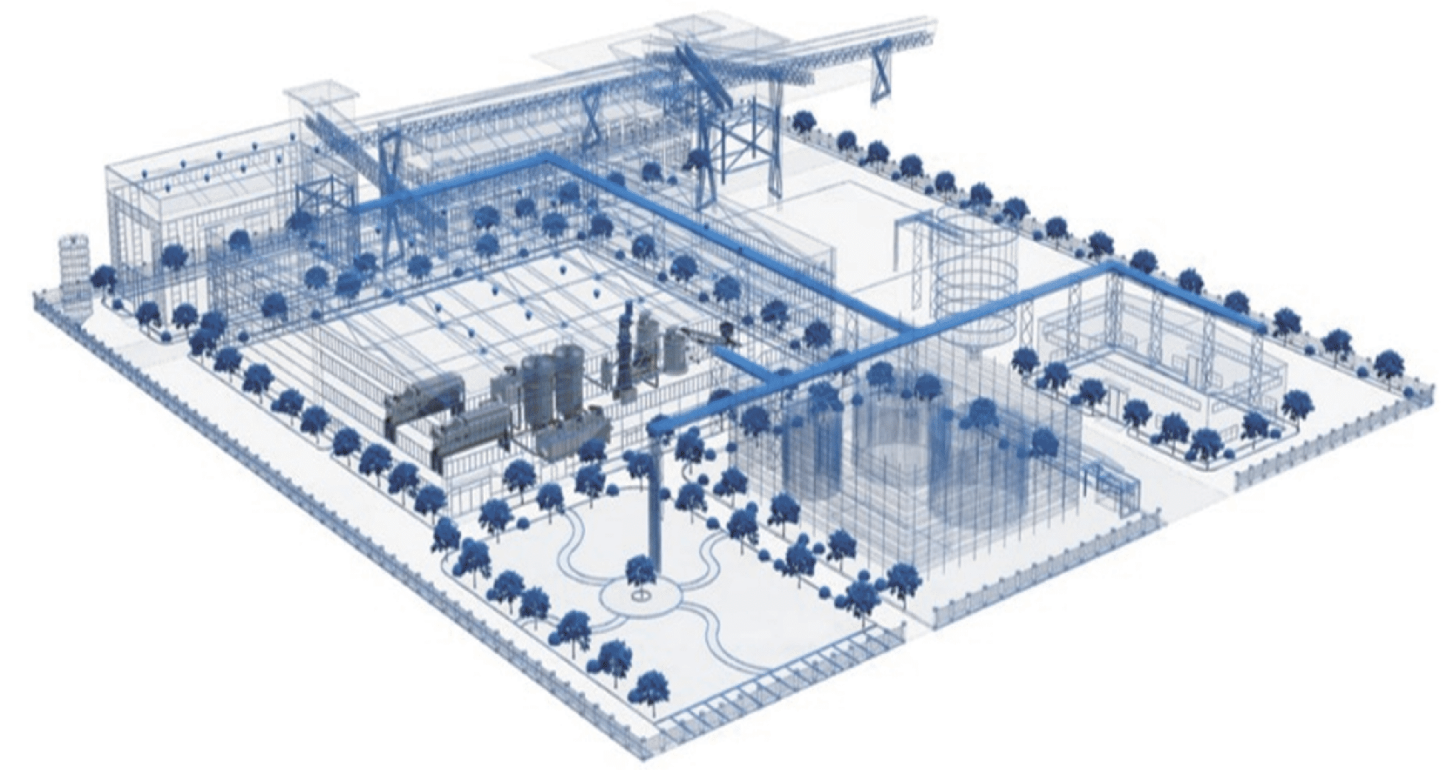

图 3 (网络版彩色)微矿分离工艺. (a) 工艺流程; (b) 25万吨/a微矿分离工业生产装置

Figure 3 (Color online) Schematic diagram of RMS process. (a) Process flow diagram; (b) layout diagram of $2.5 \times 10^{5}$ t/a industrial RMS plant

粒的SRM物流. 针对入料灰分(即“矿物质含量”)高达 55\%的原煤或者煤泥，可燃体回收率(即“碳质组分的提 取率”)可达 $83 \%$ 以上, 同时CSF产品灰分可控制在 $6 \%$ 左 右. 采用常规分离设备处理同等原料, 很难同时达到 CSF灰分低于 $7 \%$ 与可燃体回收率高于 $80 \%$ 的工艺指标. (4) 分离之后形成的CPS物流, 可脱水制成CSF, 也可以 不经脱水或部分脱水进人PLF制备单元. 在CPS物流中 加人级配颗粒群(通常粒度范围为 150 500 $\mu \mathrm{m}$ ), 通过构 造多峰分布来配制水基PLF. 此燃料的固含率可达 $65 \%$ $72 \%$ ，表观黏度为800 1200 mPa s(CSF干基热值约为 $7500 \mathrm{kcal} / \mathrm{kg}$ ). 常规水煤浆(非常普遍的工业原料、工 业燃料)的固含率为 $60 \%$ 63\%, 相比而言, PLF在气化
效率、燃烧效率方面的潜在对比优势相当大. (5) 分离 之后形成的SRM物流，先进人沉降池去除 $80 \%$ 左右的 水(此水打循环到破磨与分离单元回用), 沉降池底流经 压滤机脱水至含水量 $30 \%$ 以下．压滤后产生的SRM滤 饼可用于下游制备NMF和SRA.

利用RMS技术不仅可加工利用优质煤，也可加工 利用劣质煤、中煤、煤泥、尾煤、煤研石及其他煤基 固体废弃物, 提高煤炭利用效率, 变次为好, 变废为 宝 $^{[14 ~ 18]}$. 该技术已于 2020 年10月在山东滕州完成了 $3000 \mathrm{t} / \mathrm{a}$ 工业放大试验及示范，处理灰分 $49 \%$ 55\%的劣 质煤泥，CSF产品灰分 $4 \% \sim 7 \%$ ，可燃体回收率 $83 \%$ $87 \%$. 基于该类劣质煤泥，经微矿分离制得的SRA，施 
用于当地的土豆大田试验，经3个季度的多轮翻土再耕 验证，与当地常规种植制度相比，增产率可达 $14 \%$ $26 \%$. 年产 25 万吨PLF的微矿分离工业生产装置(图3 (b))自2020年10月开始建设, 预计2021年建成投产.

\section{2 超细颗粒解离}

我们在近期研究中发现煤炭中矿物质的解离度与 磨矿所致颗粒粒度有很明显的依赖关系，即在一定的 尺度范围, 解离度随颗粒粒度的减少而显著增加. 基于 矿物解离度分析仪(mineral liberation analyzer, MLA)对 煤炭颗粒进行矿物嵌布形态表征，发现在一定粒度范 围内分离工艺产物矿物质解离度与颗粒粒径呈明显的 线性关系，此规律未见报道. 图4为山东滕州地区一个 煤泥样品(矿物质含量为 $65 \%$ )的矿物嵌布形态表征以 及解离度分析结果. 分离过程人料的颗粒最大直径 D100在10 40 $\mu \mathrm{m}$ 范围内, CSF产物的矿物质含量与 D100呈现明显的线性关系; 而在D 100 大于 $70 \mu \mathrm{m}$ 时, $\mathrm{CSF}$ 产物的矿物质含量变化幅度较小(矿物质含量均在 $15 \%$ 以上), 相应于较低的解离度水平, 解离度不足限制 了分离工艺性能; D100小于 $10 \mu \mathrm{m}$ 的情况下, CSF矿物 质含量已小于 $2 \%$, 逐步趋近于微粒碳质组分-无机组分 解离度的极限.

由于控制出料粒度D 100 到 $40 \mu \mathrm{m}$ 以下的煤粒粉碎 成本很高，现有粉体制备工艺达到同一粒度所需的耗 电量在50 150 kW h/t开煤的水平. 这严重制约了微细 粒尺度下煤炭矿物质分离工艺的研究开发与应用. 目 前用于颗粒超细粉碎的设备可分为 3 种类型：(1) 带磨 介运动式磨机, 包括搅拌磨、振动磨、球磨等; (2) 无

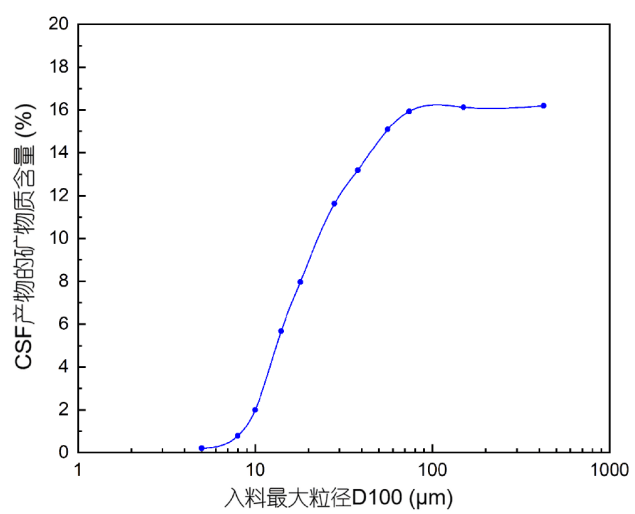

图 4 (网络版彩色)某煤泥样品在不同最大人料粒度条件下CSF产 物的矿物质含量

Figure 4 (Color online) Mineral content of CSF products by using coal slime samples with different maximum feeding particle sizes
磨介运动式磨机，包括雷蒙磨、胶体磨、高压辊磨等; (3) 冲击式磨机, 包括机械冲击磨、气流磨等. 在研磨 出料粒度D100小于 $40 \mu \mathrm{m}$ 时, 搅拌磨已被证明具有明显 的技术优势, 主要体现在设备紧凑、可长周期操作、 能耗及材料综合消耗低. 我们试制了一套新型的高效 解离磨, 采用高速剪切部件与高硬耐磨磨介, 辅以超细 颗粒表面改性剂、微粒碳质组分-无机组分优选解离 尺度表征, 在人料粒度 $0 \sim 500 \mu \mathrm{m}$ 、出料粒度 $0 \sim 40 \mu \mathrm{m}$ 条 件下的干物料耗电量在20 50 kW h/t水平, 已达到行业 先进水平. 基于颗粒破碎过程机理, 尝试建立了矩阵-粒 群平衡模型(matrix population balance model, M-PBM) 用于预测超细研磨过程的能耗，M-PBM已被证明可精 确地预测实验室规模摚拌磨机的粒度分布迁移规律和 能耗值 ${ }^{[19]}$.

此能耗水平还有进一步的改进空间，而且超细颗 粒解离领域也必须要长期探索如何显著提升电能转化 为微粒表面能的有效比例(目前这一比例为 $1 \% \sim 5 \%$ ). 在受限空间内引人超声波空化、微射流和共振等外加 场, 提高颗粒间的相互冲挤、剪切作用强度, 是未来有 望实现技术突破的一大设计理念.

\section{3 超细颗粒矿物质分离}

随着颗粒粒度不断变小，颗粒的表面活性官能团 组成、表面亲疏水特性、表面电位特性等都会发生显 著的变化. 与颗粒惯性力相比, 表面力的作用逐渐成为 主导因素. 分离工艺是否先进, 很重要的一个特征是能 否合理地分配颗粒表面改性的外场力及外部输入能 量流.

在料浆被泵入微纳分离塔之前，根据颗粒粒度及 表面特性, 决定好分离助剂的主要成分与用量; 分离助 剂的主要功能是调节固体颗粒表面的亲疏水性及电性 能, 并调节液相的表面张力. 通过剪切活化、定位空化 分两级实现微粒表面改性. 所谓剪切活化, 指的是通过 输人能量使料浆形成高度湍流，微粒表面实现更新活 化, 并使分离助剂与微粒发生充分混合. 所谓定位空 化, 其主要原理是通过调控浆体压力分布, 在液固界面 上定向产生可稳定存在的微纳米气泡. 两级微粒表面 改性的能量输人分配, 与颗粒群性质紧密相关.

经表面改性处理的料浆进入微纳分离塔后, 黏附 微纳米气泡的微粒, 经多相界面后发生选择性絮团, 碳 质组分与无机组分的絮团尺度及介观性能发生显著变 化. 可采用化工多相流流型调控手段实现碳质、无机 
颗粒的高效分离, 进而在分离塔顶获得富含碳质颗粒 的CPS物流、在塔底获得富含无机颗粒的SRM物流, 主要分离过程如图 5(a)所示. RMS技术可用于处理超细 颗粒体系, 对于此类体系, 采用现有的煤分选技术仍未 能实现工业化 ${ }^{[20 \sim 23]}$.

\section{4 类液体燃料制备}

含碳材料源经微矿分离加工后, 可得到CPS. CPS 可进一步制取清洁锅炉燃料、湿法气化原料以及发动 机燃料, 可选用的介质包括水、醇醚、油等. 液体燃料 是指可以产生热能或动力的液态可燃物质. 液态介质 中加人微细碳粒, 使得混合液具有部分液体燃料的流 动性质, 但又不是严格的液体燃料, 所以称为PLF.

PLF的不同应用在碳粒清洁度、介质、助剂、化 学转化速率、稳定性等方面的需求差异较大. 对于锅 炉、气化炉用料, 一般采用优质煤作为固体原料、水 作为介质，制成的水煤浆(固含率一般为 $60 \%$ 63\%) 就 是一种PLF. RMS技术可将劣质高灰原煤及煤基固废 加工成清洁的PLF, 以劣质、高灰原煤及煤基固废替代 优质煤, 是不可再生能源的一种高效资源化利用途径.

PLF虽然在储运、使用、安全性等方面有很大优
势, 但是燃烧与气化过程水分所需要的气化潜热占固 体燃料热值的 $5 \%$ 7\%, 提升PLF(水煤浆)的固含率是提 高系统能效非常重要的技术手段. 采用 $6500 \mathrm{kcal} / \mathrm{kg}$ 的 固体燃料制备PLF, 当固含率由 $60 \%$ 提升到 $70 \%{ }^{[24]}$, PLF 的热值由 $3684 \mathrm{kcal} / \mathrm{kg}$ 提高到 $4388 \mathrm{kcal} / \mathrm{kg}$, 增幅为 $19.1 \%$. 在CPS中加人粗颗粒与细颗粒, 辅以分散剂、 稳定剂与高速剪切混合, 提高颗粒之间的填充效率, 实 现定向分散, 进而获得清洁高浓PLF, 其制备流程如图5(b) 所示.

\section{5 天然远古矿物质用于土壤改良}

微矿分离过程制得的SRM未经表土矿物质流失与 污染、来源广泛、成本低廉, 并具有比表面积较大、 阳离子交换量高、矿质养分种类丰富、重金属含量低 等特点, 具有开发制成NMF及SRA的优势. 前期研究已 基本证明SRM可应用于无机肥料控缓释、土壤改良与 调理、生物质腐熟发酵、沙漠与矿山的土壤重构等领 域(图6). 合理利用SRM可有效缓解农业生产氮、磷、 钾大量元素利用过程中的流失, 提高肥料利用率, 促进 实现减肥增效; 同时, 可对土壤进行调理和结构重塑, 提高土壤的保墑保肥与抗侵蚀能力, 促进地力提升与
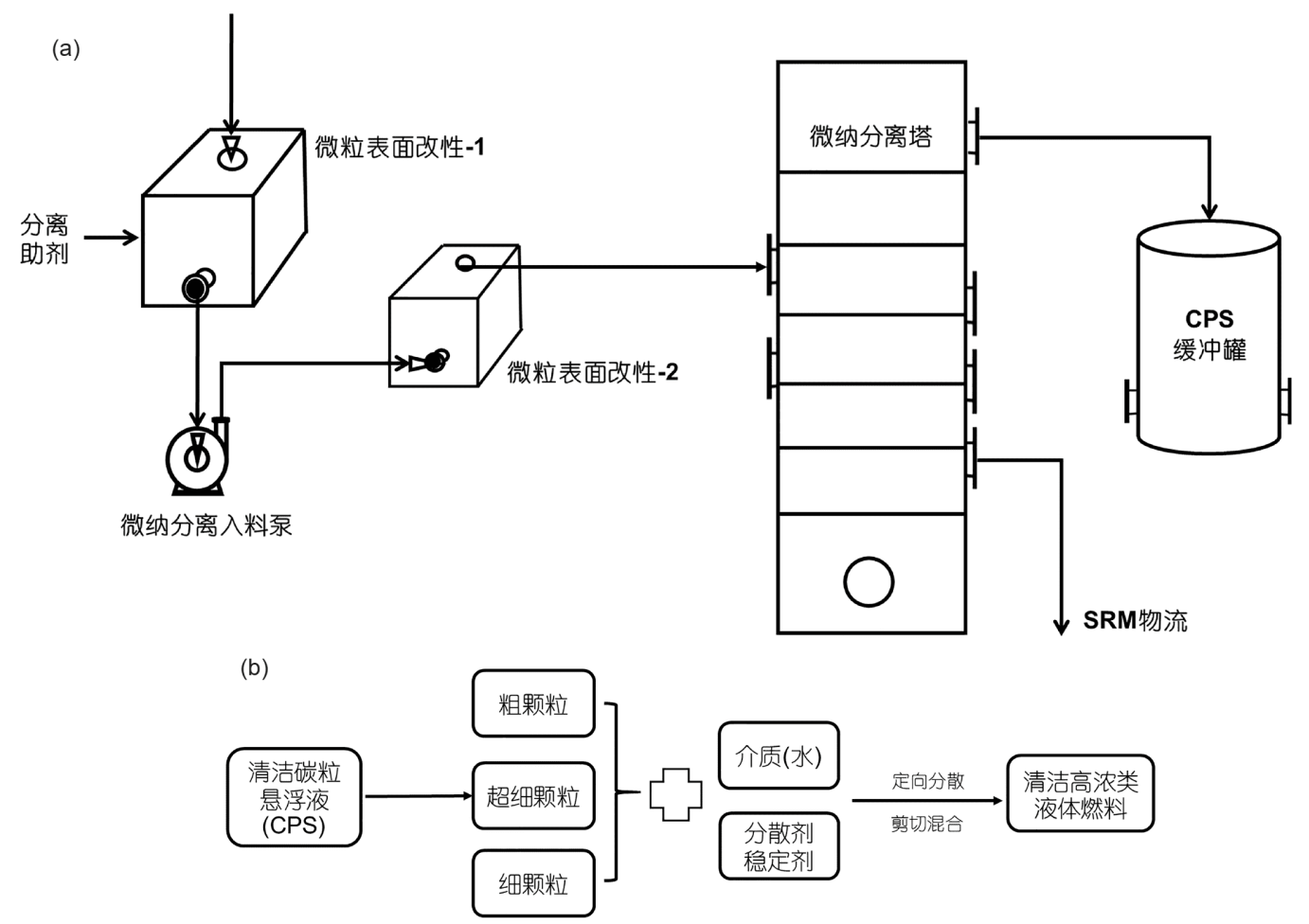

图 5 超细颗粒矿物质分离过程(a)和清洁高浓PLF制备过程(b)示意图

Figure 5 Diagram of ultrafine mineral separation process (a) and schematic diagram of clean and high concentrated PLF preparation (b) 


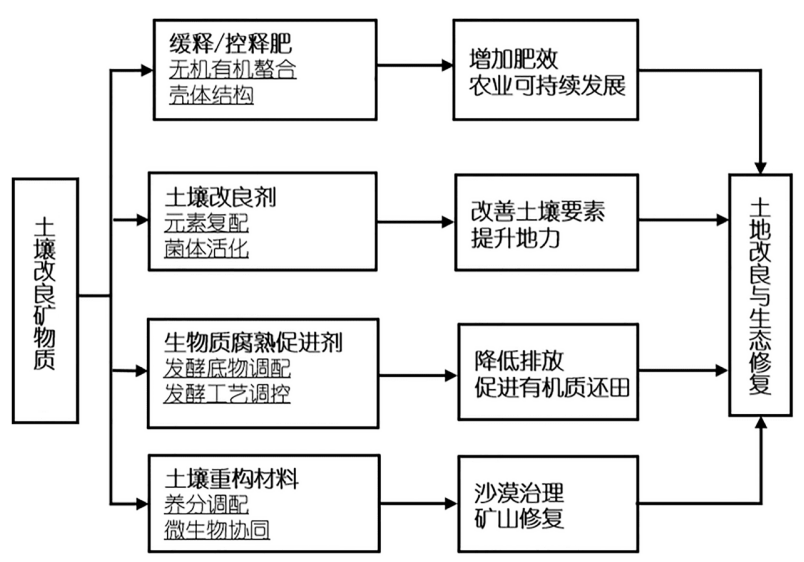

图 6 SRM应用于土壤改良与生态修复

Figure 6 Applications of SRM on soil remediation and ecological restoration

矿区生态恢复, 对保障我国土地和粮食安全具有重要 意义。

\section{2 工艺技术验证与应用分析}

\section{1 小试及工业放大}

在完成RMS小试的基础之上，开展工业放大装置 的设计与建设. 表 1 给出了RMS小试装置与工业放大装 置所处理原料的处理能力及煤质分析结果. 由表1可知, 小试实验中原料干基灰分(即灰含量)为 $55 \%$ 左右, CSF 产率为 $38 \% \sim 41 \%$ ，可燃体回收率达到 $79 \% \sim 83 \%$; 工业 放大装置中，原料干基灰分为 $50 \%, \mathrm{CSF}$ 产率为 $44 \%$ $46 \%$, 可燃体回收率达到 $83 \% \sim 87 \%$. 经由小试、工业放 大装置所制得CSF 与 $\mathrm{SRM}$ 产物的样品分析测试结果表 明：（1）小试装置中原料干基灰分为55\%左右，经过 RMS工艺处理后，获得的CSF干基灰分降低至 $7.58 \%$, 底部富集的SRM干基灰分为 $86.16 \%$; (2) 工业放大装置 中, 原料干基灰分为 $50 \%$ 左右, 经RMS工艺处理后 CSF
干基灰分降低至 $6.12 \%, \mathrm{SRM}$ 干基灰分为 $86.26 \%$.

RMS技术已由实验室的小规模工艺论证阶段迈向 半工业生产，初步实现了产学研相结合的目标. 由表 1 中数据可以看出，技术放大过程中分离性能表现出了 一致性, 从 CSF品质提升(分离助剂用量大幅下降)与可 燃体回收率整体上升, 可判断本次工艺放大已达到 预期.

长石、高岭石和绿泥石是健康土壤的重要组成矿 物. 经矿物学分析, SRM中富含上述三类矿物成分, 总 质量比例在 $50 \%$ 80\%范围. 某滕州煤泥经微矿分离加 工所得 SRM的矿物学和元素含量分析结果见表 $\mathrm{S} 1$ 和 S2. SRM可促进植物的生长, 其作用机理包括: (1) 调节 土壤结构和 $\mathrm{pH}$; (2) 提供枸溶态中微量元素; (3) 促进重 金属固定及稳定化; (4) 提高土壤固碳能力.

\section{2 技术竞争力分析}

\subsection{1 微矿分离工业项目技术经济性分析}

年产 25 万吨微矿分离工业生产装置, 联合应用 1.4 节所述PLF制备技术，合计可生产75万吨PLF产品，用 于下游大型能源化工企业用户的气化制甲醇. 而甲醇 价格受制于油价, 表 S3 给出了该项目在不同市场条件 下的技术经济性.

对比案例 $A \sim D$, 分析了国际油价在30 90美元/桶范 围内波动时项目的技术经济性. 由表可知, 内部收益率 (internal rate of return, IRR)随着国际油价的增长而降 低，当国际油价高达90美元/桶时(最近一次，2014年平 均油价为 90 美元/桶), IRR降低至 $28.92 \%$. 但敏感性分 析显示, 项目盈亏平衡点变化不大, 当生产负荷保持 $35 \%$ 时均可保证项目盈亏平衡.

案例 $\mathrm{E}$ 显示了原料品质对经济性的影响. 基准工况 中原料煤泥的干基灰分为 $50 \%$, 将原料品质调整为干基 灰分 $60 \%$ 时，IRR降低至 $14.59 \%$, 装置需要 $53.73 \%$ 的生

表 1 RMS小试和工业放大试验原料的分析测试结果 ${ }^{\text {a) }}$

Table 1 Analysis results of raw materials in a lab scale RMS test unit and another industrial scale-up RMS test unit

\begin{tabular}{cccccc}
\hline 项目 & 小试(2019年10月) & 小试(2020年9月) & 工业放大(2020年12月) & 工业放大(2020年12月) \\
\hline 处理能力 & \multicolumn{2}{c}{$6.6 \mathrm{~kg} / \mathrm{h}(50 \mathrm{t} / \mathrm{a})$} & & $500 \mathrm{~kg} / \mathrm{h}(3600 \mathrm{t} / \mathrm{a})$ \\
原料干基灰分 $(\mathrm{wt} \%)$ & 54.46 & 55.69 & 49.96 & 49.96 \\
$\mathrm{CSF}$ 产率(wt\%) & 41.17 & 37.95 & 43.96 & 46.54 \\
可燃体回收率(wt\%) & 83.06 & 79.63 & 83.39 & 86.47 \\
\hline
\end{tabular}

a) 两个小试所用的原料批次不同, 2020年9月小试所用分离助剂约为2019年10月小试的一半, 其他条件一致; 两个工业放大试验所用的原 料相同, 二者所用的分离助剂类型不同, 其他条件一致; 两个工业放大试验所采用的分离助剂量, 约为 2020 年 9 月小试的一半 
产负荷才能盈亏平衡. 目前市面上大部分的煤泥均为 干基灰分 50\%的煤泥，对于处理干基灰分 $60 \%$ 的煤泥， 需要通过技术改进实现经济性的提高.

2019年10月～2020年3月期间，突发新型冠状病毒 疫情和国际能源局势变化. 案例 $\mathrm{F}$ 和 $\mathrm{G}$ 讨论了该特殊时 期煤泥原料价格对技术经济性的影响，便于理解项目 将来遇到特殊市场环境时的适应性. 案例F中，煤泥价 格处于高位时 $(100$ 元 $/ t)$, 税后IRR为 $19.11 \%$, 盈亏平衡 点为 $46.48 \%$. 案例 $\mathrm{G}$ 中, 市场状况较为理想, 即适中的 原料价格和平均的产品价格，税后IRR为 $38.87 \%$, 盈亏 平衡点为 $30 \%$ 左右.

\subsection{2 类液体燃料浓度对甲醇生产成本的影响}

以煤制甲醇工艺流程考察PLF固含率变大后, 对单 位甲醇生产成本的影响. 当PLF固含率变大时，气化效 率提升，整个气化装置所使用的原料煤用量、氧耗量 以及用水量都相应下降; 同时, 由于进人气化炉中水量 减少, 所需的水分气化潜热显著下降, 而节省的这部分 热量可将 $\mathrm{CO}_{2}$ 转变为 $\mathrm{CO}$ 和 $\mathrm{O}_{2}$, 从而减少了 $\mathrm{CO}_{2}$ 排放.

以 180 万吨/a甲醇生产装置为例，进行技术经济性 评估. 由表S4可知，当PLF固含率由 $60 \%$ 增加至 $70 \%$ 后， 每年可节省原料煤 43 万吨 $(14.5 \%)$ ，氧耗量降低3.8亿 $\mathrm{Nm}^{3}(21.5 \%)$ ，用水量减少 89 万吨(45\%)，同时 $\mathrm{CO}_{2}$ 排放 量减少 135 万吨(32\%).

在不同PLF固含率下，甲醇生产成本随生产规模及 煤炭价格的变化如图7所示．当PLF固含率由 $60 \%$ 提升 至70\%，各条件下的甲醇生产成本普遍降低且降低幅 度较大. 同时, 在不同生产规模下甲醇生产厂对煤炭价
格波动的应对能力增强. 以年甲醇生产规模 180 万吨及 煤炭价格 500 元/t进行计算，当PLF固含率为 $60 \%$ 时，甲 醇生产成本为 1307 元 $/ \mathrm{t}$; 当PLF固含率提高到为 $70 \%$ 时， 甲醇生产成本降低至1177元/t. 故PLF固含率提升 $10 \%$ 之后，每年可为该工厂节约生产成本 2.34 亿元. 由此可 知，降低PLF原料成本、提升PLF固含率，都是增加企 业竞争性和促进产业节能减排的重要措施.

\subsection{3 微矿分离技术市场竞争力分析}

RMS技术的主要竞争力分析如下.

(1) 使用常规的商业技术和设备, 很难获得可燃体 回收率大于 $80 \%$ 的同时灰分小于 $7 \%$ 的洁净煤产品. 经 小试和工业放大试验验证，RMS工艺在CSF制备方面 具有独到优势.

（2）CSF产品替代现有固体燃料，因其高热值与低 灰分(燃烧污染主要来源)特点，将显著提高热/电生产 效率，同时可以减少煤炭燃烧过程的污染物 $\left(\mathrm{SO}_{x} / \mathrm{NO}_{x}\right.$ ' PM等)排放量，各类污染物的控制处理成本预计可降 低 30\%以上. 这将有助于有效减排和控制雾䨪，尤其是 对于中小型锅炉的减排.

(3) 与传统水煤浆相比, PLF产品固含率和能量密 度可显著提升, 进而提高气化和燃烧过程的热效率. 同 时，因为碳质颗粒中灰分可显著调低，气化、燃烧过程 的结焦、结渣等现象将显著降低，有助于核心反应装 置及管道的长周期稳定运行，可提高长期的运行效率, 运行维护成本预期可降低 $40 \%$.

(4) CSF产品可用作天然气和油品的添加剂，明显 降低燃料的综合成本，并提高了燃料热值及能量密度.

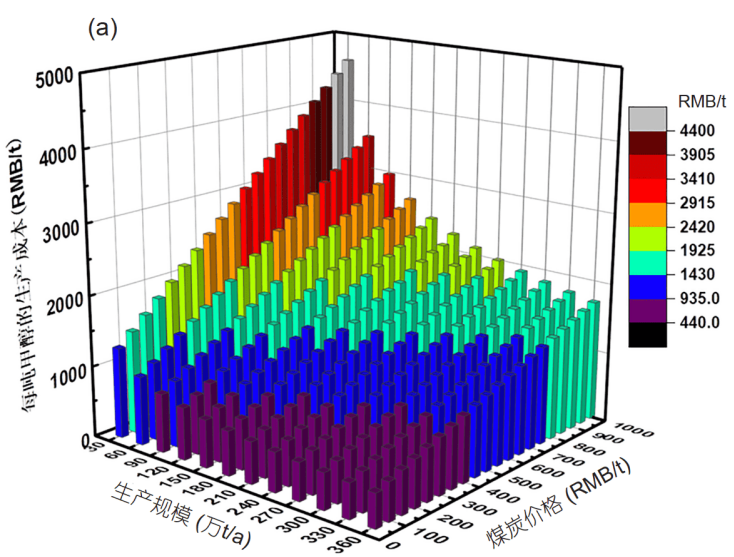

(b)

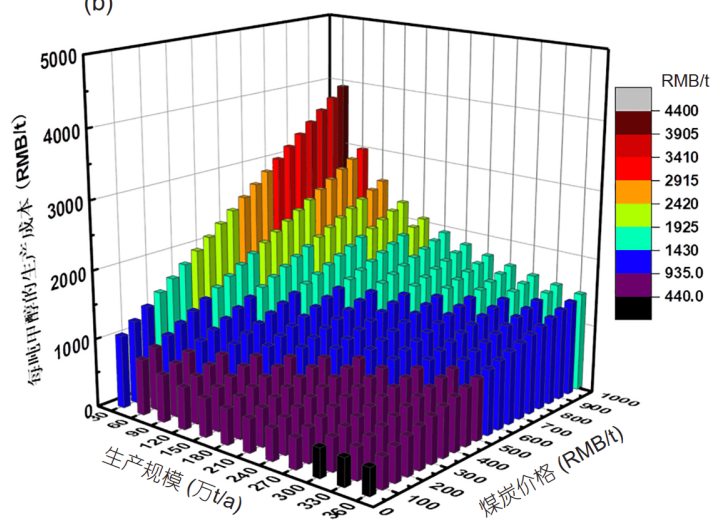

图 7 (网络版彩色)在60\%(a)和70\%(b)PLF固含率下甲醇生产成本随生产规模和煤炭价格的变化

Figure 7 (Color online) Variation of methanol production cost with production scale and coal price under solid mass fraction of PLF of $60 \%$ (a) and $70 \%(b)$ 
CSF产品可进一步通过热解、加氢等处理制得用于固 体燃料、液体燃料(如汽柴油)以及气体燃料(如天然 气)的添加剂, 降低石油和天然气宝贵资源的依赖度.

(5) 由SRM制成的SRA具有土壤改良及修复、保 肥保墒、提升农作物营养成分及产量等作用. 由SRM 制成的NMF具有肥料缓释作用，可减少肥料养分在土 壤中的损失、减少施肥作业次数及人工成本，并避免 过量施肥问题.

(6) 有效管理土壤和作物系统, 可促进 $\mathrm{CO}_{2}$ 的封存. 因为土壤中的碳储存可通过有机质分解累积与腐殖质 转化过程实现 ${ }^{[25,26]}$, 同时可通过SRA促进植物增产而 强化光合作用量实现碳封存. 所生产的SRA已被证明 可有效促进植物增产, 具有良好的固碳应用潜力. 同时 该微米级矿物进人土壤系统后, 经一系列生物化学转 化将可能产生微纳米级活性固碳矿物, 进一步提高土
壤的固碳能力. 如图8所示，使用RMS技术发电厂的 $\mathrm{CO}_{2}$ 净排放量可显著降低，主要原因包括：(1) SRM的 $\mathrm{pH}$ 为 $8.0 \sim 8.8$, 施用后可明显增强土壤的碳吸附量; (2) 由于植物增产带来碳封存增量; (3) 使用更为清洁的燃 料CSF提高了发电效率，相对降低了碳排放量. 相比之 下, 如不采用RMS技术, $\mathrm{CO}_{2}$ 将被直接释放, 无法获得任 何碳封存的补偿, 而且不得不承担大量灰渣的处理成 本和环境负担(伴有相应的 $\mathrm{CO}_{2}$ 排放). 从理论上讲, 在 森林和农业等土地应用中, 施用SRA, 可通过植物增产 $30 \% \sim 80 \%$ 而使得固碳率提高30\% 80\%. 表2提供了燃 用CSF并同时施用不同SRA的3套 $\mathrm{CO}_{2}$ 吸放量模拟预测 数据, 其中RMS装置原料的矿物质含量在 $50 \% \sim 62 \%$, $\mathrm{CSF}$ 产品产能固定为 25 万吨/a. 随着原料矿物质含量增 大, SRM产量变大且 SRA可涵盖种植总面积也随之变 大, 可达到5800 9600公顷/a. 施用SRA后, 森林系统的 (a)

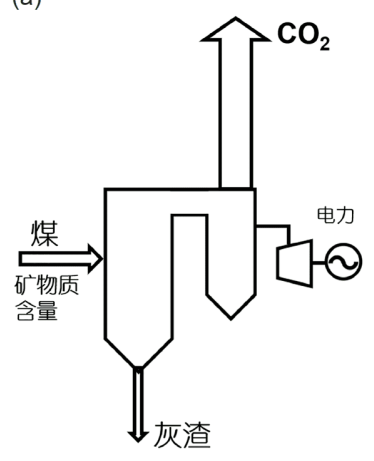

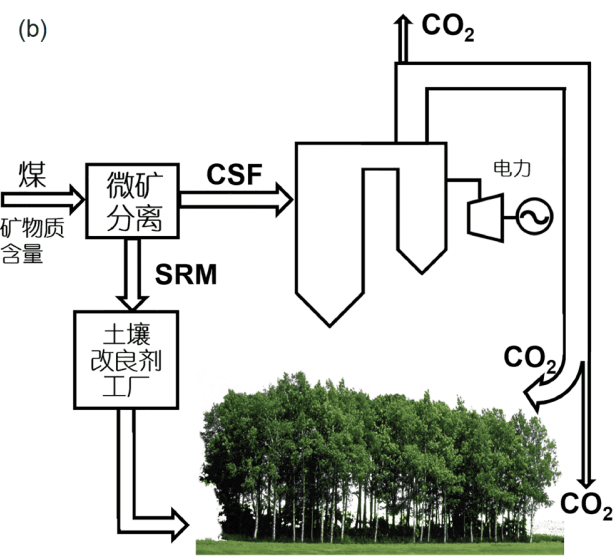

图 8 (网络版彩色)基于微矿分离技术的燃煤发电过程的 $\mathrm{CO}_{2}$ 足迹示意图. (a) 传统电厂; (b) 基于 RMS技术的电厂

Figure 8 (Color online) Schematic diagram of $\mathrm{CO}_{2}$ footprint in coal-fired power generation process based on RMS. (a) Traditional power plant; (b) power plant with RMS technology

表 2 燃用 CSF 与施用 $S R A$ 的 $\mathrm{CO}_{2}$ 吸放量估算结果 ${ }^{\text {a) }}$

Table 2 Estimation of $\mathrm{CO}_{2}$ uptake and discharge by burning CSF and applying SRA

\begin{tabular}{cccc}
\hline 原料中矿物质含量 & $50 \%$ & $56 \%$ & $62 \%$ \\
\hline $\mathrm{CSF}$ 产量(万吨/a) & 25.0 & 25.0 & 25.0 \\
$\mathrm{SRM}$ 产量(万吨/a) & 25.0 & 31.8 & 40.8 \\
$\mathrm{SRA}$ 可涵盖种植总面积(公顷/a) & 5861 & 7456 & 9566 \\
燃用CSF导致的 $\mathrm{CO}_{2}$ 排放量(万吨/a) & 69.5 & 69.5 & 69.5 \\
施用SRA后森林系统 $\mathrm{CO}_{2}$ 固化量(万吨/a) & 48.7 & 61.9 & 79.4 \\
“煤炭-电厂-森林”系统 $\mathrm{CO}_{2}$ 排放量(万吨/a) & +20.8 & +7.6 & -9.9 \\
\hline
\end{tabular}

a) 假设有效土壤厚度为 $18 \mathrm{~cm}$, 土壤容重 $1.2 \mathrm{t} / \mathrm{m}^{3}, \mathrm{SRM}$ 在有效土壤层中的平均质量分数为 $2 \%$; 假设发电厂的 $\mathrm{CO}_{2}$ 排放量为 $2.78 \mathrm{t} / \mathrm{t} \mathrm{CSF}, \mathrm{CSF}$ 碳含量为 $76 \mathrm{wt} \%$; 施用SRA后森林系统的 $\mathrm{CO}_{2}$ 固化速率基于林地固碳速率 $2.3 \mathrm{~kg} \mathrm{C} /\left(\mathrm{m}^{2}\right.$ a) 估算 ${ }^{[27]}$; “煤炭-电厂-森林”系统 $\mathrm{CO}_{2}$ 排放量的正值代表 总体排放 $\mathrm{CO}_{2}$, 负值代表总体吸收 $\mathrm{CO}_{2}$ 
$\mathrm{CO}_{2}$ 固化量预计可从 48.7 万吨/a提高到 79.4 万吨/a，“煤 炭-电厂-森林, 三元系统的 $\mathrm{CO}_{2}$ 排放量可从总体排放 $\mathrm{CO}_{2}$ 转化为总体减排 $\mathrm{CO}_{2}$. 这是基于煤炭新型利用技术 理念实现碳中和的新契机.

\section{3 技术应用前景分析}

微矿分离技术已进人工业示范阶段, 如果该技术 的低设备投资和低运行成本指标可以在工业规模装置 上得到进一步验证与规模化推广, 将深刻影响电力、 分布式能源、热力、陆运、船运、化工、农业等行业. 具体应用前景, 分析如下.

(1) 清洁能源与绿色化工应用. CSF及PLF可广泛 应用于电站锅炉、工业锅炉和工业窑炉代油、代气、 代煤燃烧, 以及宾馆、住宅、酒店、办公楼等各种建 筑物供暖、供冷和生活热水, 是洁净煤技术的重要组 成部分. 按全国散煤年消费总量约为 5 亿吨测算, 此领 域CSF及PLF产品的保守市场容量在5000万吨/a以上.

CSF及PLF可用于煤化工行业如合成氨、甲醇、 二甲醚等, 还可用于煤的直接与间接液化、以煤气化 为基础的多联产等领域, 并可大大降低煤气化联合循 环的投资和运营成本, 为这一技术的商业化应用开辟 了新途径. 煤气化作为洁净煤技术的重要组成部分具 有龙头地位, 它将廉价的煤炭转化成为清洁合成气, 增 强煤化工产品面对石油化工产品竞争的成本领先地位. 按 2020 年煤化工原料市场容量 2 亿吨测算, 此领域CSF 及PLF产品的保守市场容量在 2000 万吨/a以上.

CSF可用于提高汽柴油、煤油、燃料油、醇醚、 天然气等各种高性价比燃料的热值和能量密度. 可广 泛用于航运、海运、陆运、电力、热力、军事装备等 行业, 提高燃烧效率、续航能力和设备动力.

(2) 土壤改良与绿色农业应用. 中国的农业生产普 遍存在过量施肥问题, 目前中国主要粮食产区的化肥 施用量为世界平均水平的3 4倍. 减肥增效是中国农业 实现可持续发展的重要途径.

将SRM进行表面处理、微生物活化、生化调控, 在鳌合剂的作用下, 与现有肥料复合可制得富含中微 量元素及矿物质的新型肥料NMF, 包括控释肥料与缓 释肥料. 通过不同的调控手段, 有效控制肥料养分释放 速率和释放期, 有效降低施肥量, 提高肥料当季利用率. 据2019年中国化肥施用折纯量为 5403.59 万吨测算, 此 领域NMF产品的保守市场容量在500万吨/a以上.

我国有机固体废弃物的肥料化是环境保护和农业
生产的双重需求. 有机物料腐熟效率、臭气及温室气 体减排、有害物质的固定化和减量化(重金属与抗生 素)是影响有机固废肥料化的关键问题. 基于矿物、有 机质与微生物三者间的交叉协同作用, 通过将SRM与 有机质联合共发酵可有望促进以上关键问题的解决.

SRM通过有机质耦合调控、功能菌剂激活和靶向 养分复配技术可制备矿物复合高效菌剂的新型有机肥 NMF及土地复垦用调理剂SRA, 可用于调节土壤团聚 体结构、提高土壤有机质水平、补充土壤有益微生物 与中微量元素. 同时, 可增加土壤的黏结性以达到减少 水土流失、修复退化土壤、促进地区生态恢复的目的. 据2019年有机肥实际产能为 3300 万吨测算, 此领域 NMF及SRA产品的保守市场容量在 330 万吨/a以上.

(3) 土地复垦与生态修复. 截至2017年底, 中国矿产 资源造成土地破坏面积为 5440 万亩 (1亩=666.667 $\mathrm{cm}^{2}$ ), 其中破坏面积较大的省份为山西、内蒙古、河北、山 东、新疆. 地貌重塑、土壤重构、植被重建、微生物 多样性恢复是矿区土地复垦与生态修复的关键技术.

SRM比表面积大、黏性大, 经表面处理、微生物 活化和生化调控, 可作为土壤重构基质用于矿区土地 复圼. 利用所得天然中微量元素及矿物质为土壤提供 养分, 并利用其良好的黏结性和保水性提高待修复土 壤的保墒能力及抗风蚀能力. 将土壤评价、土壤重 构、生态修复、景观规划等环节有机系统结合, 利用 植物土壤反馈体系的调控机制, 实现采矿区土地、严 重退化土地以及沙漠的综合治理. 按国家与地方的土 地复星、城乡建设用地指标置换管理办法, 可利用复 垦后的工矿废弃地进行城乡建设用地指标置换, 这将 有效缓解严守耕地红线与建设用地紧缺的矛盾, 具有 良好的社会效益和经济效益.

\section{3 结论}

针对煤炭及煤基固废等含碳材料源, 基于矿物材 料纳米尺度解构、超细颗粒微米尺度解离、多相流表 界面改性与调控等理念提出了一种新型的矿物分离工 艺技术, 即RMS技术. 该技术可有效地将含碳材料源中 的微量元素及矿物质分离出去. 围绕RMS技术, 开展了 深人的基础研究、工艺开发和放大试验, 测试了 20 种 以上不同的含碳材料源, 在山东滕州已建成了工业放 大试验及示范装置. 这项技术从原理上不同于其他煤 炭加工技术, 具有良好的技术竞争力与市场竞争力. 通 过该技术可制备CSF、PLF和SRM等主要产品. 
CSF及PLF可用作燃料或材料的替代品, 在电力/热 力、钢铁冶金、煤气化/液化以及煤化工等行业具有很 好的应用前景. 由于其低灰和高热值等特点, 将显著提 高生产过程效率, 降低运行维护成本, 同时可以有效减 少污染物排放量. RMS技术可实现煤中可燃组分与无 机矿物的高效分离，这对于合理利用煤炭资源、改善 环境污染问题同时改良土壤都具有重要意义.

SRM经一系列的物理化学和生化处理，可制成富 含有益矿物质及有机质的复合型肥料和SRA. 将其用 于土壤改良与修复, 预期可促进土壤健康, 有助于提高 食物链中的营养成分含量和均衡程度. 近期研究结果 表明，施用新型SRA可以显著提高作物产量. 经 3 个季
度的多轮翻土再耕验证，与当地土豆常规种植制度相 比，增产率可达 $14 \%$ 26\%. SRM应用技术的开发与实 践，将在化肥减量增效、有机废弃物资源化利用、土 地复旺与生态修复、沙漠治理等方面产生重要的影响。 SRM的使用可以大幅提高植物产量及光合作用量，以 植物形式固定更多的 $\mathrm{CO}_{2}$, 结合 $\mathrm{CSF}$ 的高效燃烧及单位 热值 $\mathrm{CO}_{2}$ 减排, 可显著减少煤炭利用过程中的 $\mathrm{CO}_{2}$ 排 放量.

RMS技术已取得了显著的进展，产业前景非常可 观，但仍有很多科学和技术问题待进一步研究和探索. 这一新技术的持续研究开发与实践应用，将为煤炭清 洁化与土壤改良带来新契机.

\section{参考文献}

1 Power Statistics and Data Center of China Electric Power Union. Operation Profile of Electric Power Industry from January to November 2020 (in Chinese). 2020 [中电联电力统计与数据中心. 2020年1 11月份电力工业运行简况. 2020]

2 Liu K. To solve the problems of dispersed coal and diesel engines is critical for haze treatment (in Chinese). Global Times, 2015-04-08 [刘科. 治䨪 先治散烧煤和柴油机. 环球时报, 2015-04-08]

3 Institute of Resources and Environmental Policy, Development Research Centre of the State Council. 2017 China Bulk Coal Treatment Research Report (in Chinese). 2017 [国务院发展研究中心资源与环境政策研究所. 2017中国散煤治理调研报告. 2017]

4 Liu K. The major root causes of smog in China and technologies and solutions to reduce it. Front Eng, 2016, 3: 343-348

5 Le T, Wang Y, Liu L, et al. Unexpected air pollution with marked emission reductions during the COVID-19 outbreak in China. Science, 2020, 369: $702-706$

6 National Development and Reform Commission, National Energy Administration. The 13th Five-Year Plan for Coal Industry Development (in Chinese). 2016 [国家发展改革委, 国家能源局. 煤炭工业发展“十三五”规划. 2016]

7 Li H J. Comprehensive Utilization of Coal Gangue (in Chinese). Beijing: Chemical Industry Press, 2010 [李化建. 煤矸石的综合利用. 北京: 化学 工业出版社, 2010]

8 Gruber K. Agrobiodiversity: The living library. Nature, 2017, 544: S8-S10

9 Marler J B, Wallin J R. Human Health, the Nutritional Quality of Harvested Food and Sustainable Farming Systems. Nutrition Security Institute White Paper, 2006

10 Wang S, Zhang Y, Ju W, et al. Recent global decline of $\mathrm{CO}_{2}$ fertilization effects on vegetation photosynthesis. Science, 2020, 370: 1295-1300

11 Arnold B J. Coal formation. In: Osborne D, ed. The Coal Handbook: Towards Cleaner Production. Cambridge: Woodhead Publishing, 2013

12 Wang G, Bai X, Wu C, et al. Recent advances in the beneficiation of ultrafine coal particles. Fuel Process Technol, 2018, 178: 104-125

13 Hu S, Liu L, Yang X, et al. Influence of different dispersants on rheological behaviors of coal water slurry prepared from a low quality coal. RSC Adv, 2019, 9: 32911-32921

14 Zhou W, Liu L, Zhou B, et al. Electrokinetic potential reduction of fine particles induced by gas nucleation. Ultrasons Sonochem, 2020, 67: 105167

15 Chen Y, Hu S, Li J, et al. Improvement on combustible matter recovery in coal slime flotation with the addition of sodium silicate. Colloid Surf APhysicochem Eng Asp, 2020, 603: 125220

$16 \mathrm{Hu}$ S, Li J, Yang X, et al. Improvement on slurry ability and combustion dynamics of low quality coals with ultra-high ash content. Chem Eng Res Des, 2020, 156: 391-401

$17 \mathrm{Hu}$ S, Chen Y, Wu C, et al. The performance and dispersing mechanism of anionic dispersants in slurries prepared by upgraded coal. Colloid Surf A-Physicochem Eng Asp, 2020, 606: 125450

18 Zhao X, Tang Y, Zhao B, et al. Collecting behaviors of high internal phase (HIP) emulsion in flotation of ultrafine high-ash content coal slime. Int J Coal Prep Util, 2021, 1-21

19 Chu C Q, Zhao B L, Bai X T, et al. Study on ultrafine pulverized coal based on matrix PBM (in Chinese). J China Coal Soc, 2019, 44: 1906-1914 [楚长青, 赵冰龙, 白雪涛, 等. 基于矩阵PBM的煤粉超细粉碎过程研究. 煤炭学报, 2019, 44: 1906-1914]

20 Evans G M, Atkinson B W, Jameson G J. The Jameson cell. In: Matis K A, ed. Flotation Science and Engineering. New York: Marcel Dekker, Inc., 
1995. 331-363

21 Li B, Tao D, Ou Z, et al. Cyclo-microbubble column flotation of fine coal. Sep Sci Technol, 2003, 38: 1125-1140

22 Jameson G J. New directions in flotation machine design. Miner Eng, 2010, 23: 835-841

23 Zhang H, Liu J, Wang Y, et al. Cyclonic-static micro-bubble flotation column. Miner Eng, 2013, 45: 1-3

24 Hu S, Jiang F, Zhao B, et al. The enhancement on rheology, flowability, and stability of coal water slurry prepared by multipeak gradation technology. Energy Fuels, 2021, 35: 2006-2015

25 Kimble J M, Heath S, Birdsey R A, et al. The Potential of U.S. Forest Soils to Sequester Carbon and Mitigate the Greenhouse Effect. Boca Raton: CRC Press, 2002

26 Lal R. Carbon sequestration in soils of central Asia. Land Degrad Dev, 2004, 15: 563-572

27 Zhang Q Y, Lü W Y, Xu B Q. Carbon sequestration capacity of urban green space in North China (in Chinese). Environ Prot Sci, 2021, 47: 41-48 [张青云, 吕伟娅, 徐炳乾. 华北地区城市绿地固碳能力测算研究. 环境保护科学, 2021, 47: 41-48]

\section{补充材料}

表S1 某SRM产品的主要矿物组成

表S2 某SRM产品的相关养分指标含量

表S3 微矿分离制清洁类液体燃料工业项目的技术经济性分析

表S4 类液体燃料提浓前后的年消耗与排放预测值

本文以上补充材料见网络版csb.scichina.com. 补充材料为作者提供的原始数据, 作者对其学术质量和内容负责. 


\title{
A novel mineral separation process: New opportunity for clean coal utilization and soil remediation
}

\author{
Changning Wu ${ }^{1,2,3}$, Li Weng ${ }^{1,2}$, Junguo $\mathrm{Li}^{1,2,3^{*}}$, Haolin Jia ${ }^{1}$, Xin Wang ${ }^{1}$, Jiali Chen ${ }^{1}$, Wei Huang ${ }^{1}$, \\ Shunxuan $\mathrm{Hu}^{1}$, Daofan $\mathrm{Cao}^{1}$, Baonan $\mathrm{Zhou}^{1}$, Changqing $\mathrm{Chu}^{2}$, Binglong $\mathrm{Zhao}^{3}$ \& $\mathrm{Ke} \mathrm{Liu}^{1,2,3^{*}}$ \\ ${ }^{1}$ Department of Chemistry, Southern University of Science and Technology, Shenzhen 518055, China; \\ ${ }^{2}$ Clean Energy Institute, Academy for Advanced Interdisciplinary Studies, Southern University of Science and Technology, Shenzhen 518055, China; \\ ${ }^{3}$ Shenzhen Engineering Research Center for Coal Comprehensive Utilization, School of Innovation and Entrepreneurship, Southern University of \\ Science and Technology, Shenzhen 518055, China \\ * Corresponding authors, E-mail: lijg@sustech.edu.cn; liuk@sustech.edu.cn
}

China currently consumes nearly 4 billion tons of coal annually with $64.7 \%$ of China's electricity in 2019 being sourced from coal-firing power plants. There are about 700000 small and medium-sized coal-burning boilers which consume 750 million tons of coal annually in 2017. These boilers are one of the major sources of air pollution and smog in China. The cost for equipping each small and medium-sized boiler with desulfurization and denitrification facilities (such as selective catalytic reduction, SCR) is prohibitively high. Additionally, it is not feasible to convert most of these coal-fired boilers to burn natural gas as the natural gas supply in China equates to about 220 million tons of standard coal units. Therefore, the only viable option for reducing air pollution while retaining energy security is to make the coal cleaner cost effectively prior to combustion. Also the introduction of chemical fertilizers (like ammonium nitrate) stimulated greater yield of crops, but changed the subsurface chemistry of the soil structure, locking up calcium, burning out humus, causing acidic soils and destroying the microorganism/mineral balance. A novel mineral separation technology has been developed. This technology (hereafter called "RevTech minerals separation technology" or "RMS technology") combines milling, separation, particle grading and soil remediation, enhanced by nanotechnology and advanced materials science with an innovative product conditioning system in energy and chemical engineering field. It effectively separates minerals and trace elements from carbonaceous feedstocks, producing high-grade and low-emission fuels, namely clean solid fuel (CSF) and pseudo liquid fuel (PLF), and natural ancient minerals called soil remediation minerals (SRM). The technology mainly involves four process steps: (1) Particles are milled using low-power consumption micronization technology, based on mineralogical characteristics; (2) carbonaceous and mineral particles are separated after specified surface modification and multiphase flow interphase regulation with proprietary reagents; (3) carbonaceous particulate flow is prepared to CSF by de-watering or pumped to PLF preparation unit, while mineral particulate flow is prepared to SRM by de-watering too; (4) the carbonaceous particulate flow is used to produce a high solid concentration PLF via multi-peaking gradation. RMS technology has been well demonstrated in a lab-scale test unit and another industrial scale-up test unit. The dry basis ash content (Ad) of the raw materials is about $55 \%$. In the laboratory scale test unit, Ad of the CSF obtained is reduced to $7.58 \%$, and Ad of the SRM enriched at the bottom is $86.16 \%$ using RMS technology process. In the industrial scale-up test unit, Ad of the raw materials is about $50 \%$. Ad of the CSF is reduced to $6.12 \%$, and Ad of SRM is $86.26 \%$ using RMS process. The calculation results indicated that when the solid mass fraction of PLF increased from $60 \%$ to $70 \%$, the production cost of methanol could be saved by 234 million $\mathrm{CNY} / \mathrm{a}$ and the $\mathrm{CO}_{2}$ emission could be decreased by $1.35 \mathrm{Mt} / \mathrm{a}$ for a $1.8 \mathrm{Mt} / \mathrm{a}$ methanol production plant. A $250 \mathrm{kt} / \mathrm{a}$ RMS commercial plant is under construction. This project has been evaluated to have good technical and market competitiveness. SRM is used to produce natural mineral fertilizer (NMF) and soil remediation amendment (SRA), offering potential solutions for soil remediation and topsoil restoration. SRM comprises feldspar, kaolinite and chlorite that are important components of healthy soils, at the total content of $50 \%-85 \%$. The SRM derived products could increase vegetation productivity, strengthen plant \& soil carbon sequestration, and achieve carbon neutrality. RMS technology could provide cost-effective clean fuels, and realize the resource utilization of coal-derived solid wastes. It provides a reliable way for the efficient and clean utilization of coal and $\mathrm{CO}_{2}$ emission reduction.

mineral separation, clean solid fuel (CSF), pseudo liquid fuel (PLF), soil remediation minerals (SRM), carbon neutrality 